\title{
SUPERVIVENCIA EN RELACIÓN A LOS FACTORES PRONÓSTICOS EN UNA SERIE DE PACIENTES CON CARCINOMA DE CÉLULAS RENALES
}

José Miguel Giménez Bachs, Mํa José Donate Moreno, Antonio S. Salinas Sánchez, Héctor Pastor Navarro, Pedro Carrión López, José María Pastor Guzmán, Lorenzo Polo Ruíz, Mariano Martínez Martín, Rafael Ruíz Mondéjar y Julio A. Virseda Rodríguez.

Servicio de Urología. Complejo Hospitalario Universitario de Albacete. Albacete. España.

\begin{abstract}
Resumen.- OBJETIVO: Analizar la supervivencia de una serie de pacientes diagnosticados de carcinoma de células renales durante un periodo de 19 años en base a los factores pronósticos utilizados habitualmente en la práctica clínica.
\end{abstract}

MÉTODOS: Estudio retrospectivo sobre 259 pacientes diagnosticados de manera consecutiva durante los años 1988 a 2006 carcinoma de células renales, que fueron tratados quirúrgicamente en nuestro servicio. A partir de los datos clínicos y patológicos y de seguimiento se ha hecho un estudio de supervivencia comparando el impacto de los factores pronósticos habituales: estadio, tamaño tumoral, grado nuclear, etc.

RESULTADOS: Se realizaron 264 cirugías a los 259 pacientes de la muestra que presentaron una edad media de 61,91 años. El diagnóstico se realizó más frecuen- temente por hallazgo incidental (52,12\% de los casos), practicándose un $72,97 \%$ de nefrectomías radicales frente a un $26,25 \%$ de parciales. El carcinoma de células claras fue el diagnóstico histológico más frecuente $(69,88 \%)$. Presentaron peor supervivencia los pacientes con carcinoma de células claras, los tumores sintomáticos, con mayor tamaño y grado nuclear de Fuhrman. A mayor estadio tumoral el pronóstico fue peor, especialmente en los tumores con estadio superior a pT3a. La presencia de ganglios linfáticos afectados o metástasis a distancia presentan una supervivencia cáncer-específica mucho más baja. La de supervivencia cáncer-específica global a los 5 años fue superior al 80\%.

CONCLUSIÓN: Los factores pronósticos usados clásicamente para predecir la supervivencia del cáncer renal siguen siendo útiles, en especial el estadio patológico pT. Se observa una mejor supervivencia en comparación con series más antiguas, pero este tipo de tumores sigue generando una morbi-mortalidad importante.

Palabras clave: Carcinoma de células renales, supervivencia, factores pronósticos.

Summary.- OBJECTIVES: To analyze the survival rate in a series of patients with the diagnosis of renal cell carcinoma over a 19 year period based on prognostic factors usually employed in clinical practice.

METHODS: Retrospective study of 259 consecutive patients with the diagnosis of renal cell carcinoma undergoing surgery in our department between 1988 and 2006. From clinical, pathological, and follow-up data we performed a survival study comparing the impact of usual prognostic factors: stage, tumor size, nuclear grade, etc. 
RESULTS: 264 surgical procedures were performed in 259 patients, with a mean age of $61.91 \mathrm{yr}$. The most frequent way of diagnosis was incidental finding $152.12 \%$ of the cases); radical nephrectomy was performed in $72.97 \%$ of the cases in comparison with $26.25 \%$ partial nephrectomies. Clear cell carcinoma was the most frequent histological diagnosis (69.88\%). Patients with clear cell carcinoma, symptomatic tumors, bigger size, and greater Fuhrman nuclear grade presented worse survivals, mainly in tumors with stage $>$ pT3a.

The presence of involved lymph nodes or distant metastases is associated with a much lower cancer-specific survival. Global five-year cancer-specific survival was over $80 \%$.

CONCLUSIONS: The classical prognostic factors used to predict survival in renal cancer are still useful, mainly pathological stage pT. We observed a better survival in comparison with older series, but this kind of tumors continue generating important morbidity-mortality.

Keywords: Renal cell carcinoma. Survival. Prognostic factors.

\section{INTRODUCCIÓN}

Dentro de la patología oncológica urológica, el Carcinoma de Células Renales (CCR) representa el tumor más letal, llegando a doblar la mortalidad por esta causa frente a la producida por los tumores vesicales o prostáticos (1). A esto hay que sumar el aumento de la incidencia de este tipo de tumor en los últimos años (2), aunque se ha producido una mejora en la supervivencia, llegando a ser superior al $60 \%$ a los 5 años actualmente, en contraposición a los años sesenta, en los que era inferior al $40 \%(3,4)$.

Este aumento de incidencia se puede explicar por el aumento en la detección de tumores en estadios más bajos, aunque la tasa de mortalidad se ha visto afectada de manera negativa, lo que hace pensar también en cambios en los factores de riesgo (tabaco, dieta o exposición a distintos agentes carcinógenos) $(2,5)$.

La nefrectomía es el tratamiento estándar del CCR y a partir de su instauración como patrón oro en el manejo de estos tumores en los años sesenta, es cuando se ha ido mejorando la supervivencia en los pacientes que padecen esta enfermedad (6), si bien se han ido caracterizando mejor los factores pronósticos clásicos y se empiezan a establecer otros nuevos que ayudan a comprender el comportamiento de estos tumores.
El presente estudio pretende analizar la supervivencia en los pacientes diagnosticados de CCR en nuestro centro a lo largo de 19 años, contrastando ésta con los distintos parámetros clínicopatológicos usados habitualmente como factores pronósticos.

\section{SUJETOS, MATERIAL Y MÉTODO}

Estudio retrospectivo observacional sobre 259 pacientes consecutivos diagnosticados y tratados por CCR durante el periodo que comprende desde enero de 1988 hasta diciembre de 2006 en el Complejo Hospitalario Universitario de Albacete.

A partir de las historias clínicas se recogieron los datos socio-demográficos y clínico-patológicos, así como del seguimiento de estos pacientes, incluyendo las causas que llevaron a la muerte, cuando ésta se produjo.

El análisis estadístico se realizó con el programa informático SPSS 13.0.1, efectuándose análisis descriptivo y análisis de supervivencia con el método de Kaplan-Meyer y finalmente con la regresión multivariante de Cox.

\section{RESULTADOS}

Se han realizado un total de 264 cirugías por cáncer renal a 259 pacientes. La edad media de los mismos fue de 61,91 años (D.E: 12,32; I.C $95 \%=$ $60,40-63,42$ años).

El $64,9 \%$ de los pacientes fueron varones, con una relación hombre / mujer de 1,85/1.

El seguimiento medio de los pacientes fue de 40,63 meses (D.E: 46,85; I.C 95\%: 34,90-46,37 meses).

La evolución en el número de diagnósticos de carcinoma renal a lo largo del periodo de estudio se muestra en la Figura 1.

El diagnóstico se realizó más frecuentemente por hallazgo incidental, lo que ocurrió en el 52,12\% de los casos, mientras que el síntoma principal más frecuente que llevó al diagnóstico de tumor renal fue la hematuria $(23,55 \%)$, seguido del dolor $(12,74 \%)$, masa $(4,25 \%)$ y el resto fueron diagnosticados por otros síntomas. Tan sólo en el $0,77 \%$ de los pacientes la clínica que llevó al diagnóstico fue la triada de Guyon (hematuria, dolor y masa palpable). 


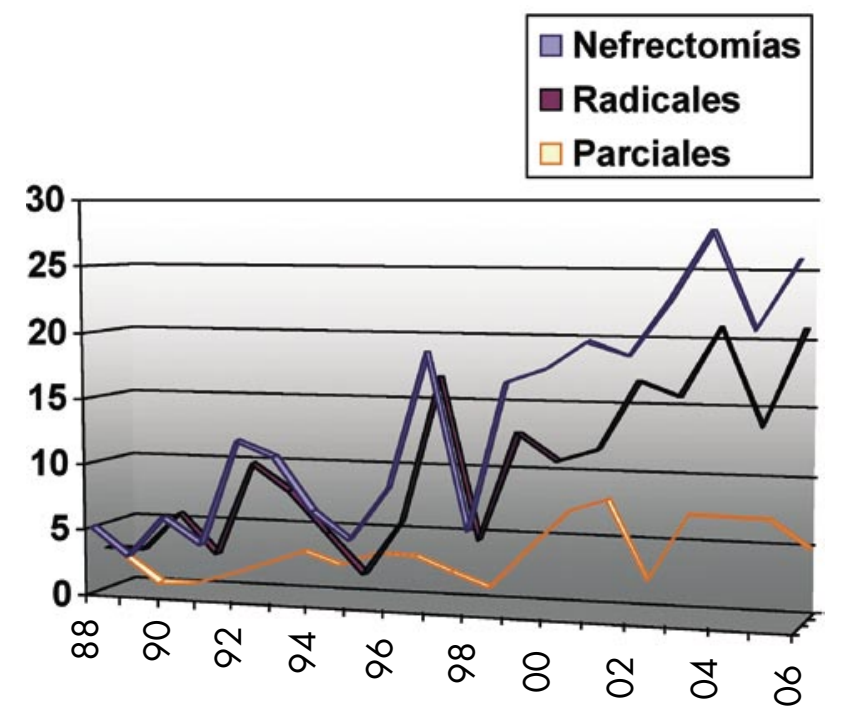

FIGURA 1. Número de nefrectomías realizadas por año indicando el número de cirugías radicales y conservadoras de parénquima.

Se practicaron un $72,97 \%$ de nefrectomías radicales, de las cuales el $41,70 \%$ fueron vía transperitoneal, $57,14 \%$ vía lumbar, mientras que un $26,25 \%$ de los pacientes fueron sometidos a cirugía conservadora de parénquima. Dos pacientes se consideraron irresecables y tan sólo se recogió biopsia tumoral en el acto quirúrgico. En 5 pacientes hubo que realizar una segunda cirugía, bien por bilateralidad o por bordes quirúrgicos afectados que obligaron a la nefrectomía radical tras una parcial.

En los pacientes en los que se realizó cirugía conservadora de parénquima, la causa más frecuente fue electiva, practicada en el $85,29 \%$ de los pacientes, mientras que se realizó por insuficiencia renal, ser monorreno o presentación de tumor bilateral en el $7,35 \%, 2,94 \%$ y $2,94 \%$ respectivamente. En un caso de realización de nefrectomía parcial la causa fue el diagnóstico de enfermedad de von Hippel-Lindau de la paciente.

El diagnóstico histológico de los tumores extirpados fue mayoritariamente de células claras $(69,88 \%)$, seguido en frecuencia del carcinoma cromófilo o papilar tal y como se muestra en la Figura 2.

El grado de Fuhrman de los tumores fue de 2 en un $55,87 \%$, seguido del grado 3 en el $27,53 \%$ y del 4 en el 10,12\%. El 4,63\% de las piezas tumorales presentaron un grado de Fuhrman no valorable. El tamaño tumoral fue en $42,08 \%$ de los pacientes entre 4 y $7 \mathrm{~cm}$. y en el $37,07 \%$ mayor de $7 \mathrm{~cm}$., siendo el resto menores de $4 \mathrm{~cm}$.

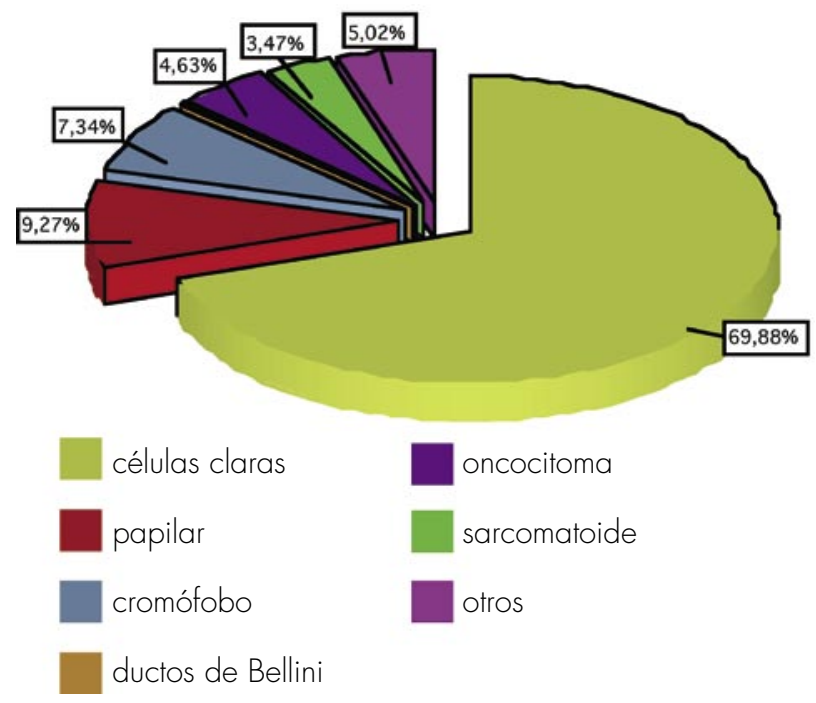

FIGURA 2. Distribución de los tumores según su tipo histológico.

En la Figura 3 se muestran los porcentajes en cuanto al estadio tumoral tras la realización de la cirugía, apreciándose que el estadio más frecuente era el estadio I. Se realizó linfadenectomía en el 16,22\% de los pacientes, siendo esta positiva (N1 ó N2) en el $35,71 \%$.

El 15,06\% de los pacientes presentaron metástasis a distancia y la localización más frecuente de las mismas fue pulmonar, en el $46,15 \%$ de los mismos, seguida de ósea en el 2,51\%. Otras localizaciones fueron en glándula suprarrenal, hígado, y en lugares más inusuales como piel.

La supervivencia tumor-específica en relación con los síntomas de presentación fue significa-

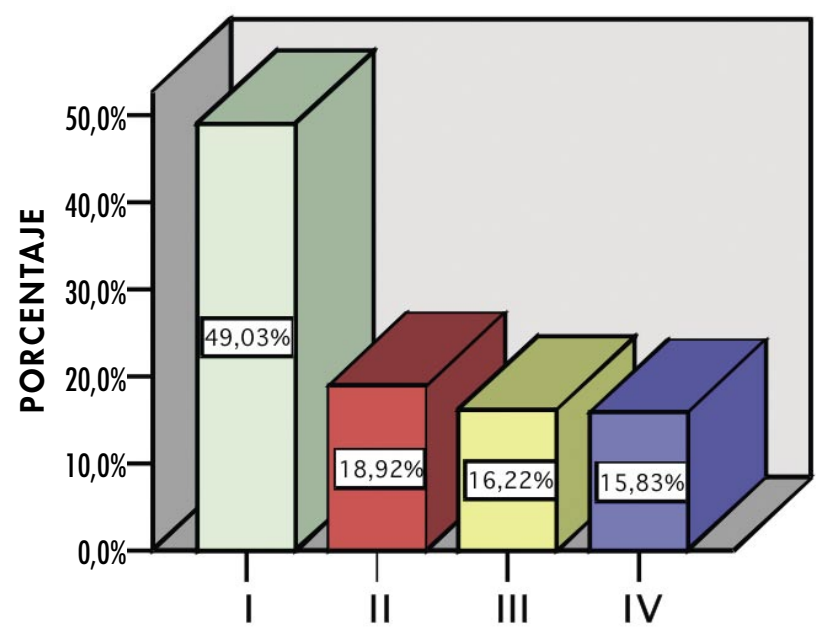

FIGURA 3. Estadio tumoral según clasificación TNM. 

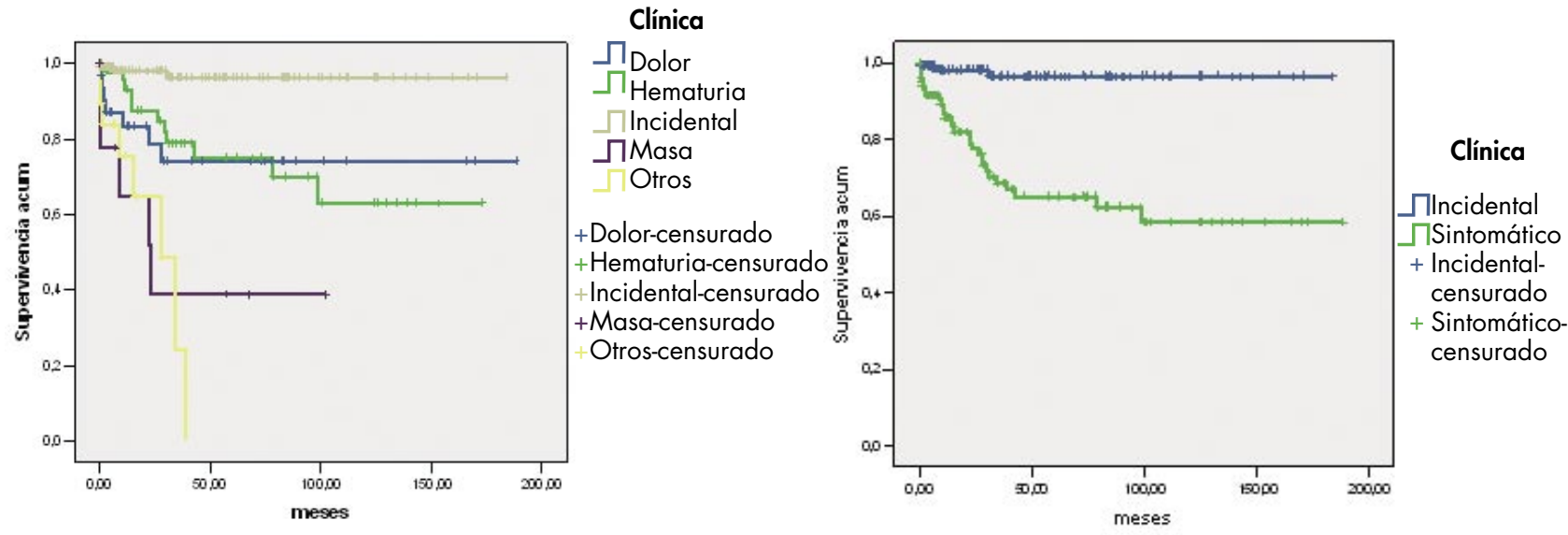

FIGURA 4. Supervivencia cáncer-específica según la clínica de presentación.

tivamente mayor $(p<0,005)$ en aquellos pacientes diagnosticados de manera incidental, teniendo peor pronóstico los catalogados en otros síntomas, que habitualmente se debían a manifestaciones metastásicas del cáncer renal. En la Figura 4 se muestra la curva de supervivencia tanto en función de cada uno de los síntomas diagnosticados, como en la división de tumores sintomáticos frente a los diagnosticados de manera incidental.

En relación a la supervivencia tumor-específica teniendo en cuenta el tipo histológico tumoral, se aprecia que diferenciando los tipos histológicos, los más letales son los inclasificados (codificados como "otros") y los que presentaban diferenciación sarcomatoide $(p<0,005)$ (Figura 5). Comparamos la supervivencia según los tumores sean o no de células claras, observando como a los 5 años la supervivencia es ligeramente mayor en los tumores de células claras $(81,74 \%)$ frente a los que no son de células claras $(78,51 \%)$, aunque con más seguimiento las supervivencias se igualan, quedando por debajo en los tumores de células claras $(77,34 \%)$ frente al otro grupo $(78,51 \%)$ a partir de los 8 años de seguimiento, aunque estos datos no son estadísticamente significativos $(p=0,165)$.

Con el grado de Fuhrman, la supervivencia empeora conforme aumenta el grado, de manera que los grado 1 no presentaron mortalidad tumor-específica en el periodo de este estudio, mientras que la supervivencia a los 5 años de los grado 4 fue del $36,62 \%(p<0,05)$. Agrupando el grado de Fuhrman en dos grupos (grados 1 y 2 frente a grados 3 y 4 ), las diferencias de supervivencia también son significativas $(p<0,05)$. Estos datos se ilustran en la Figura 6.

Otro factor estudiado en la supervivencia tumor-específica es el tamaño tumoral. En la Figura 7 se observa que los tumores presentaron peor superviven- cia conforme aumentaban su tamaño, de manera que los tumores menores de $4 \mathrm{~cm}$. presentaron una supervivencia a los 5 años del $94,91 \%$, frente al $87,93 \%$ y $65,91 \%$ de los que presentaron un tamaño entre 4 y $7 \mathrm{~cm}$ y los mayores de $7 \mathrm{~cm}$ respectivamente.

La supervivencia en relación al estadio pT demuestra de manera significativa $(p<0,005)$ que conforme aumenta el estadio pT empeora la supervivencia. Destaca que los tumores pT2 y los tumores pT3a tienen supervivencias a los 5 años parecidas $(81,29 \%$ y $76,35 \%$ respectivamente), pero empeora

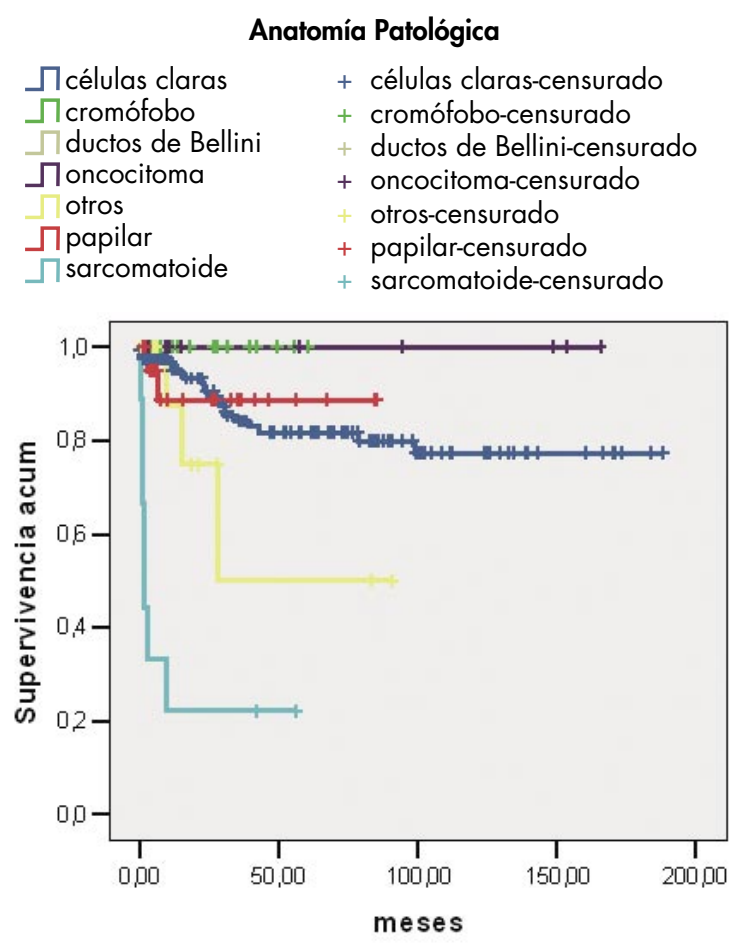

FIGURA 5. Supervivencia cáncer-específica atendiendo al tipo histológico tumoral. 

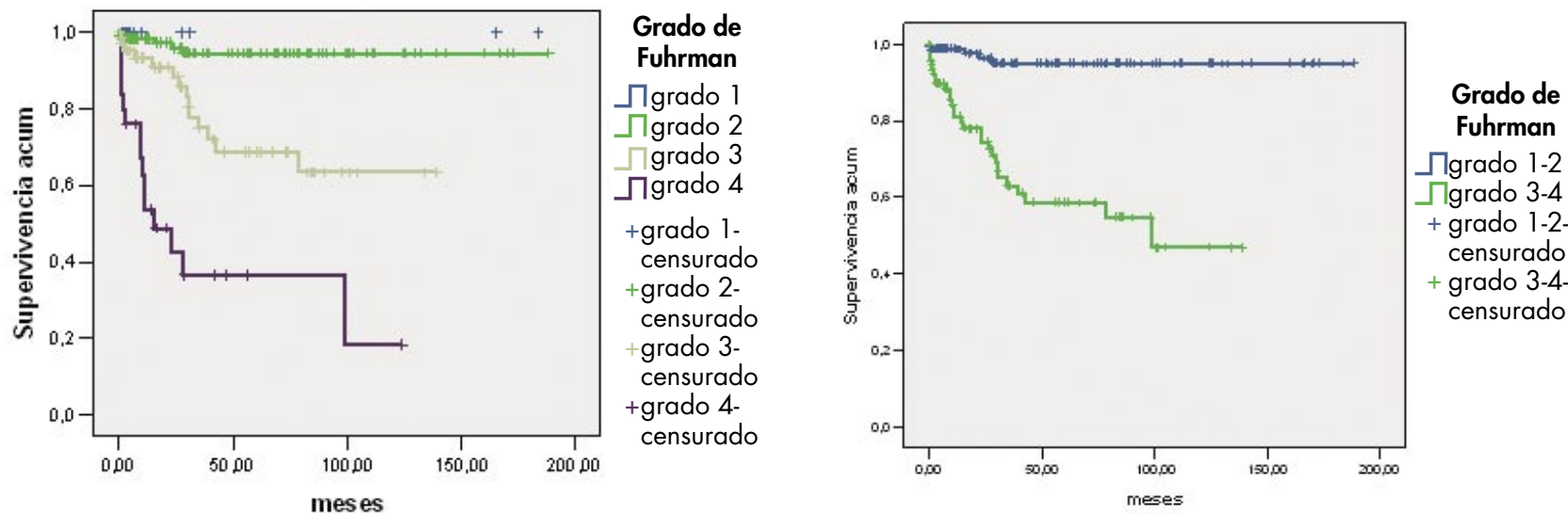

FIGURA 6. Supervivencia cáncer-específica en relación al grado de Fuhrman por grados y agrupado.

de manera importante en estadios más avanzados (Figura 8).

$\mathrm{Si}$ atendemos a la supervivencia tumor-específica relacionada con la presencia de afectación ganglionar, se observa que los de los pacientes que presentaron ganglios positivos (sólo se realizó linfadenectomía a 42 pacientes, de los cuales 15 presentaron afectación ganglionar) presentaron una pobre supervivencia en comparación con los que tenían los ganglios libres $(p<0,05)$ (Figura 9).

De los pacientes que presentaron metástasis a distancia, sólo 3 de ellos $(1,17 \%)$ presentaron supervivencia por encima de 5 años, y tan solo uno de ellos permancía vivo al finalizar este estudio. Estos resultados se reflejan en la Figura 10.

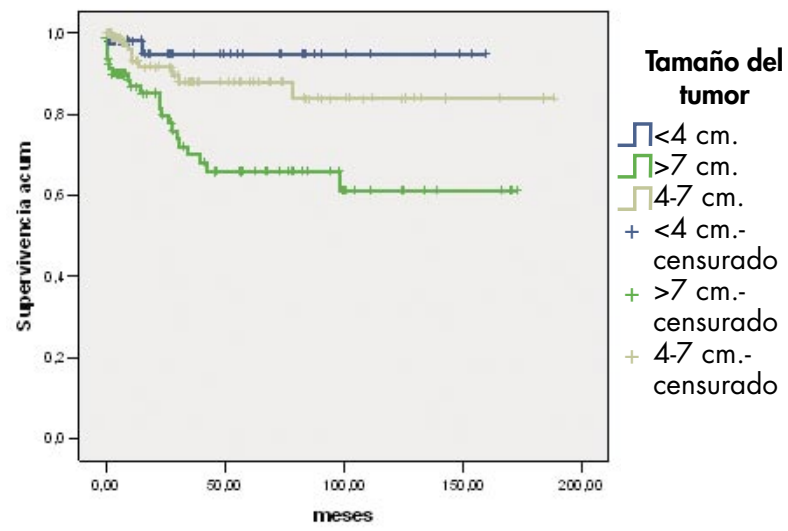

FIGURA 7. Supervivencia cáncer-específica por tamaño tumoral.
La curva de supervivencia cáncer-específica global se muestra en la Figura 11. Se observa una supervivencia a los 5 años superior al $80 \%$. En el análisis de regresión de Cox, el único factor que permaneció como variable independiente dentro del modelo con significación estadística fue el estadio tumoral $(p<0,001)$.

\section{DISCUSIÓN}

El patente incremento en la incidencia de los tumores renales puede ser explicado en parte por el uso generalizado de técnicas de imagen tales como la ecografía, lo que, por otra parte permite el diagnóstico precoz que a su vez da paso a la práctica de técnicas quirúrgicas menos agresivas y con resul-
Estadio pT

ПpTla
$П$ pTlb
$\Pi$ pT2
$\Pi$ pT3a
pT3b
$П$ pT3c
$\Pi$ pT4
+ pTla-
censurado
+ pT1b-
censurado
+ pT2-
censurado
+ pT3a-
censurado
pT3b-
censurado
+ pT3c-
censurado
+ pT4-
censurado

FIGURA 8. Supervivencia cáncer-específica según el estadio pT. 

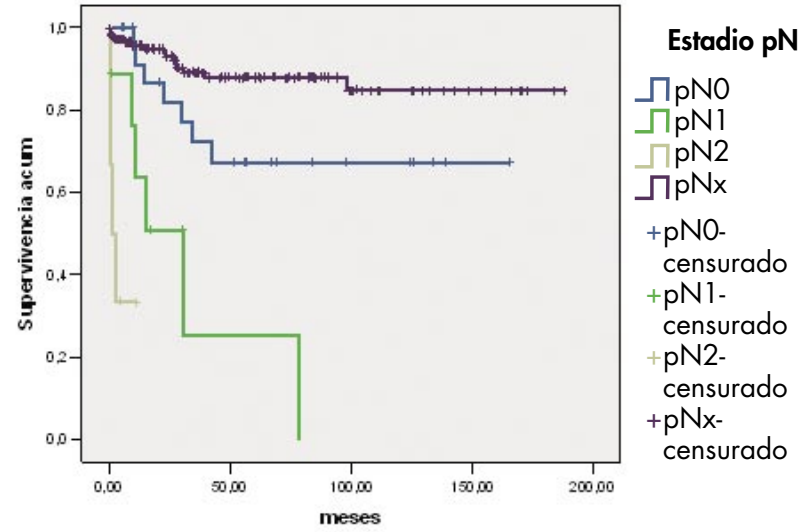

\section{FIGURA 9. Supervivencia cáncer-específica según el estadio $\mathrm{pN}$.}

tados similares $(2,7,8)$. Este aspecto queda reflejado en nuestra serie, ya que se aprecia un aumento progresivo del diagnóstico de tumores renales desde finales de los ochenta hasta la actualidad, así como un incremento en la práctica de cirugías conservadoras.

La proporción de los distintos subtipos histológicos permanece más o menos constante en comparación con lo descrito en la literatura (9), de manera que los tumores de células claras siguen siendo la variante más común, seguidos del subtipo papilar. Cabe destacar un leve aumento en el diagnóstico de los tumores cromófobos (por encima del $5 \%$ ), lo que concuerda con otros estudios que ya habían detectado esta tendencia (10).

El estadio patológico, habitualmente establecido por la clasificación TNM, es el mayor determinante para predecir el pronóstico en pacientes con $C C R$, de manera que suele ser uno de los factores más importantes a la hora de proponer un tratamiento. No obstante, parece haber una discrepancia entre estadio clínico y patológico, con tendencia a la supraestadificación, lo que debemos de tener en cuenta a la hora de decidir si practicamos cirugía conservadora de parénquima o cirugía radical (11). Existe cierta controversia en la clasificación TNM con respecto a los estadios $T 1$ y $T 2$, ya que considera únicamente el tamaño, y parece que no hay diferencias significativas, en términos de supervivencia entre lo tumores entre 4 y $7 \mathrm{~cm}$ (estadio $\mathrm{Tl}$ b) y los mayores de $7 \mathrm{~cm}$ (estadio II) (12), aunque este aspecto debe tomarse con precaución a la hora de decidir cirugía conservadora (13).

Por el hecho, ya comentado anteriormente, del aumento del uso de técnicas de imagen, los tumo-

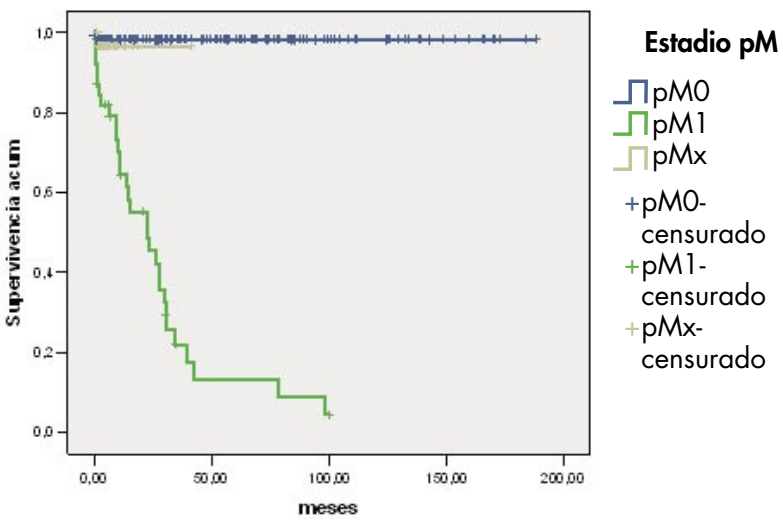

FIGURA 10. Supervivencia cáncer-específica según el estadio $\mathrm{pM}$

res renales se diagnostican cada vez más en estadios más precoces, y por tanto, la mayoría de las veces cuando todavía no han presentado ninguna sintomatología, lo que les confiere un mejor pronóstico (14). En el presente estudio más de la mitad de los pacientes fueron diagnosticados de manera incidental, $y$ es en éstos donde se aprecia una mejor supervivencia, mientras que la hematuria es el tercer síntoma con peor pronóstico después de los pacientes con manifestaciones metastásicas o en los que el tumor era palpable. Por tanto, el hecho de que el diagnóstico sea incidental supone un dato a tener en cuenta a la hora de establecer el pronóstico de estos pacientes.

Al analizar la supervivencia en relación al subtipo histológico de los tumores, el carcinoma de

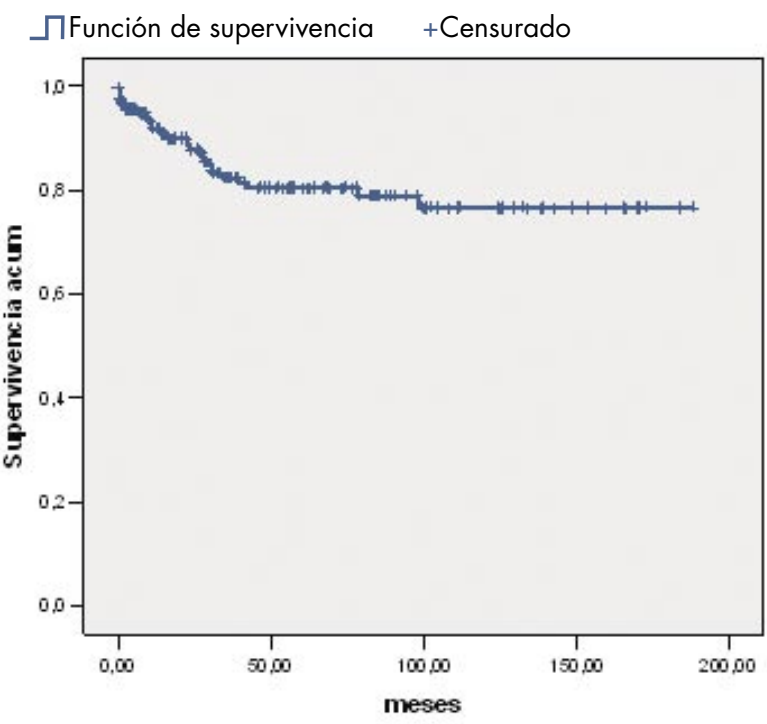

FIGURA 1 1. Supervivencia cáncer-específica global. 
células claras sigue siendo el de peor pronóstico (10), si no tenemos en cuenta aquéllos que sufrieron diferenciación sarcomatoide, que por sí ya muestra empobrecimiento de la supervivencia (15), y los tumores catalogados en este estudio como "otros", habitualmente inclasificables, por no cumplir los criterios de los demás subtipos, bien por su arquitectura mixta, por presentar células irreconocibles o por mostrar mezcla de componentes estromales y epiteliales (16). Se ha visto que tumores con arquitectura predominantemente papilar muestran componentes celulares típicos del carcinoma de células claras e incluso en su caracterización molecular (17). Por esta razón, el subtipo más frecuente (células claras) es además el que peor pronóstico presenta. Al analizar la supervivencia de los tumores de células claras frente al resto, no obtuvimos significación estadística, quizá explicado por estar en el otro grupo los inclasificables y los que presentaron diferenciación sarcomatoide, siendo este hecho más frecuente también en los tumores de células claras (9).

El grado nuclear de Fuhrman empeora el pronóstico conforme aumenta, si bien es un factor que ha sido criticado por su subjetividad. No se han encontrado diferencias pronósticas entre los grados 1 y 2 y se ha propuesto un sistema nuevo que una ambos grados, ya que parece ser que la influencia pronóstica del grado nuclear es más significativa cuando se agrupan los tumores bien diferenciados (grados 1 y 2 de Fuhrman) y los tumores indiferenciados (grados 3 y 4) $(15,18)$. En nuestra serie se comprueba esta tendencia, tanto si obtenemos las curvas de supervivencia con los grados nucleares aislados o agrupados en dos grupos.

La participación del tamaño en la supervivencia de los pacientes con CCR es clara. Conforme aumenta el tamaño el pronóstico empeora, y es muy probable que la causa sea también porque los tumores detectados de manera incidental habitualmente son de menor tamaño y por tanto los pacientes presentan mejores supervivencias (14).

Se discute si la clasificación de los estadios pT1 y pT2 es adecuada, puesto que parece que el punto de corte más apropiado para el tamaño tumoral es de $5 \mathrm{~cm}(15,19)$ ya que no parece haber una diferencia importante en cuanto a la supervivencia de los pacientes con estadio pT1 y pT2. En cuanto al estadio pT3, parece ser que el subestadio pT3a no implica peor pronóstico, y que los tumores así clasificados tienen un comportamiento similar en cuanto a recidivas y supervivencia que los estadios inmediatamente inferiores, por lo que se les puede considerar como tumores órgano-confinados $(20,21)$. El resto de estadios presentan un pronóstico más desfavorable, aunque la suma de otros factores pronósticos tales como tamaño, grado nuclear y presencia de metástasis ganglionares o a distancia son los que empeoran de manera significativa la supervivencia en estos pacientes (22).

El papel de la linfadenectomía en el CCR no está del todo claro, no se ha demostrado un aumento de la supervivencia y no hay un acuerdo sobre cuál debe ser su extensión y la morbilidad al realizar esta técnica puede aumentar considerablemente (23). Lo que sí parece claro es que la invasión de los ganglios linfáticos es un indicador de un mal pronóstico con un significativo impacto sobre la supervivencia (24).

Las metástasis suponen un factor determinante en la supervivencia de los pacientes con CCR. Si bien en el presente estudio parece que hay un menor porcentaje de lo descrito en otros trabajos (25), el impacto sobre la supervivencia es definitivo (23).

La supervivencia global en pacientes con CCR parece mejorar en los últimos años (3), y el presente estudio así lo confirma. No obstante seguimos necesitando establecer más factores pronósticos que nos ayuden a predecir de manera más fiable el comportamiento de los tumores renales. De momento podemos decir que el estadio tumoral continua siendo el parámetro más fiable (11), pero debemos sumarle otros elementos determinantes en el pronóstico, ya hablemos de supervivencia como de progresión o recidiva, tales como tamaño, grado nuclear, tipo histológico, etc. que pueden servir para predecir la conducta de esta enfermedad (26).

\section{CONCLUSIÓN}

El CCR sigue representando una patología con importante impacto en la morbimortalidad de los pacientes y los factores pronósticos definidos clásicamente continúan siendo útiles de cara al tratamiento, en espera que vayan apareciendo nuevas herramientas diagnósticas y terapéuticas que permitan un enfoque más óptimo para el manejo de estas neoplasias.

\section{BIBLIOGRAFÍA y LECTURAS

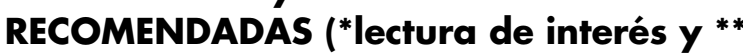 lectura fundamental)}

**1. NOVICK, A.C.; CAMPBELL, S.C.: "Renal Tumors". Campbell's Urology, 8th Edición, Walsh PC, Retik AB, Vaughan DE, Wein AJ. Pág. 26722731. Edit. Saunders Company Philadelphia (Pennsylvania), USA, 2003. 
*2. GIMÉNEZ BACHS, J.M.; DONATE MORENO, M.J.; SALINAS SÁNCHEZ, A.S. y cols.: "Incidencia creciente en el carcinoma de células renales". Actas Urol. Esp., 30: 295, 2006.

*3. McLAUGHLIN, J.K.; LIPWORTH, L.: "Epidemiologic aspects of renal cell cancer". Semin. Oncol., 27: 115, 2000.

**4. PANTUCK, A.J.; ZISMAN, A.; BELLDEGRUN, A.S.: "The changing natural history of renal cell carcinoma". J. Urol., 166: 1611, 2001.

*5. CHOW, W.H.; DEVESA, S.S.; WARREN, J.L. y cols.: "Rising incidente of renal cell cancer in the United Status". JAMA, 281: 1628, 1999.

6. FICARRA, V.; MARTIGNONI, G.; LOHSE, C. y cols.: "External validation of the Mayo Clinic stage, size, grade and necrosis (SSIGN) score to predict cancer specific survival using a european series of convencional renal cell carcinoma". J. Urol., 175: 1235, 2006.

7. PANTUK, A.J.; ZISMAN, A.; RAUCH, M.K. y cols.: "Incidental renal tumors". Urology, 56: 190, 2000.

8. GIMÉNEZBACHS, J.M.; SALINAS SÁNCHEZ, A.S.; HERNÁNDEZ MILLÁN, I.R. y cols.: "Cirugía conservadora de parénquima en los tumores renales". Rev. Clin. Esp., 204: 191, 2004.

*9. STÖRKEL, S.; EBLE, J.N.; ADLAKHA, K. y cols.: "Classification of renal cell carcinoma. Workgroup $\mathrm{N}^{\circ} 1$. Union Internationale contre le cancer (UICC) and the American Join Committee on Cancer (AJCC)". Cancer, 80: 987, 1997.

10. REUTER, V.E.; PRESTI, J.C.: "Contemporary approach to the classification of renal epithelial tumors". Semin. Oncol., 27: 124, 2000.

*11. SVATEK, R.S.; LOTAN, Y.; HERMANN, M. y cols.: "The influence of clinical and pathological stage discrepancy on cancer specific survival in patients treated for renal cell carcinoma". J. Urol., 176: 1321, 2006.

**12. STEINER, T.; KNELS, R.; SCHUBERT, J.: "Prognostic significance of tumor size in patients after tumor nephrectomy for localised renal cell carcinoma". Eur. Urol., 46: 327, 2004.

13. PATARD, J.J.; SHVARTS, O.; LAM. J.S. y cols.: "Safety and efficacy of partial nephrectomy for all T1 tumors based on an international multicenter experience". J. Urol., 171: 2181, 2004.
14. TSUI, K.H.; SHVARTS, O.; SMITH, R.B. y cols.: "Renal cell carcinoma: Prognostic significance of incidentally detected tumors". J. Urol., 163: 426, 2000.

*15. ALGABA, F.; ARCE, Y.; TRIAS. I. y cols.: “Aplicación clínica de las actuales clasificaciones del cáncer renal". Actas Urol. Esp., 30: 372, 2006.

**16. BOSTWICK, D.G.; EBLE, J.N.: "Diagnosis and classification of renal cell carcinoma". Urol. Clin. North Am., 26: 627, 1999.

17. SALAMA, M.E.; WORSHAM, M.J.; DEPERALTA-VENTURINA, M.: "Malignant papillary renal tumors with extensive clear cell change". Arch. Pathol. Lab. Med., 127: 1176, 2003.

18. GOLDSTEIN, N.S.: "Grading of renal cell carcinoma”. Urol. Clin. North Am., 26: 637, 1999.

19. FICARRA, V.; GUILLÉ, F.; SCHIPS, L. y cols.: "Proposal for revision of the TNM classification system for renal cell carcinoma". Cancer, 104: 2116, 2005.

20. GILBERT, S.M.; MURPHY, A.M.; KATZ, A.E. y cols.: "Revaluation of TNM staging of renal cortinal tumors: Recurrence and survival for T1NOM0 and T3aNOM0 tumors are equivalent". Urology, 68: 287, 2006.

21. GÓMEZ PÉREZ, L.; BUDÍA ALBA, A.; PONTONES MORENO, J.L. y cols.: "Evaluación del estudio pT3a de la actual clasificación TNM del cáncer renal". Actas Urol. Esp., 30: 287, 2006.

22. SÁNCHEZ SALABARDO, D.; AROCENA GARCÍA-TAPIA, J.; REGOJO BALBOA, J.M. y cols.: "Factores pronósticos en carcinoma renal pT3". Actas Urol. Esp., 27: 26, 2003.

*23. RINI, B.I.; VOGELZANG, N.J.: "Prognostic factors in renal cell carcinoma". Sem. Oncol., 27: 213, 2000.

24. VAN BRUSSEL, J.P.; MICKISCH, G.H.J.: "Prognostic factors in renal cell and bladder cancer". BJU Int., 83: 902, 1999.

25. LANG, H.; JACQMIN, D.: "Prognostic factors in renal cell cancer". EAU Update Series, 1: 215, 2003.

*26. SORBELLINI, M.; KATTAN, M.W.; ZINDER, M.E. y cols.: "A postoperative prognostic nomogram predicting recurrence for patients with convencional clear cell renal cell carcinoma". J. Urol., 173: 48, 2005. 